\title{
The Associations between Anger Expression and Insomnia among Community-Dwelling Older Adults in Korea
}

\author{
See Woo Kim¹, Kyung Mee Park1, Woo Jung Kim², Eun Chae Choi', Suk Kyoon An', \\ Kee Namkoong ${ }^{1}$, Yoosik Youm³ ${ }^{3}$ Hyeon Chang Kim', and Eun Lee ${ }^{1}$ \\ 'Department of Psychiatry and Institute of Behavioral Science in Medicine, Yonsei University College of Medicine, Seoul, Korea \\ 2Department of Psychiatry, Myongji Hospital, Goyang, Korea \\ ${ }^{3}$ Department of Sociology, Yonsei University College of Social Sciences, Seoul, Korea \\ ${ }^{4}$ Department of Preventive Medicine, Yonsei University College of Medicine, Seoul, Korea
}

\begin{abstract}
Objective: Sleep disturbance is one of the most common health problems in older adults. Anger expression tendency has been studied on many aspects of health. Some studies have reported that individuals with poor anger regulation were more likely to have insomnia. Considering emotional arousal affects insomnia, anger expression tendency could be associated with inaomnia in older adults. The objective of this study is to investigate correlation between anger expressions and insomnia symptoms among community-dwelling older adults in Korea. Methods: A total of 235 older adults (mean age 75 years, 67.2\% female) were enrolled to this study. We interviewed the participants to gather demographic information, medical history, stress level using the Perceived Stress Scale, sleep quality using the Pittsburgh Sleep Quality Index and anger expression using Korean adaptation of the State-Trait Anger Scale. Simple and logistic regression analyses were used to find correlations between anger expression and insomnia symptoms. Results: Forty percent of the participants had sleep initiation difficulty (sleep latency $>30 \mathrm{~min}$ ). Higher anger expression tendency (odds ratio $=1.122, \mathrm{p}=0.009$ ) was significantly related to sleep initiation difficulty after controlling age, sex, education, socioeconomic status, perceived stress, depression, and total sleep time. Meanwhile, anger expression tendency was not associated with total sleep time after controlling covariates. Conclusion: Older adults with higher anger expressions showed a tendency to have sleep initiation difficulty. Further studies are needed to determine whether modifying of anger expression can control physiological and cognitive arousal and improve sleep disturbance.
\end{abstract}

Key Words: Insomnia; Sleep; Sleep latency; Sleep initiation dysfunction; Anger

Received: April 1, 2019 Revised: May 22, 2019 Accepted: May 24, 2019

Corresponding author: Eun Lee, MD, PhD, Department of Psychiatry and Institute of Behavioral Science in Medicine, Yonsei University College of Medicine, 50-1 Yonsei-ro, Seodaemun-gu, Seoul 03722, Korea.

Tel: 82-2-2228-1620, Fax: 82-2-313-0891, E-mail: leeeun@yuhs.ac

(a) This is an Open Access article distributed under the terms of the Creative Commons Attribution Non-Commercial License (https://creativecommons.org/licenses/bync/4.0) which permits unrestricted non-commercial use, distribution, and reproduction in any medium, provided the original work is properly cited.

\section{INTRODUCTION}

Sleep disturbance is one of the most common health problems in older adults. They often experience more sleep disturbance than young adults. The most commonly diagnosed sleep disorder among the elderly is insomnia [1]. Insomnia is defined as having symptoms related to sleep including difficulty initiating or maintaining sleep, nocturnal waking, and waking without feeling rested. Prevalence as well as severity of insomnia is known to increase with age [2]. Insomnia prevalence rate among older adults is similar across the world despite some differences depending on measure- ment tools. In the United States, $20-30 \%$ of the older adults are known to experience insomnia [3], and 28\% in the African older adults. In Korea, it is reported that 29-40\% incidence of insomnia is found in the study of community-sample older adults [4].

One of the main reasons for the high prevalence of insomnia among older adults is associated with ageing. Ageing is associated with alteration of circadian cycle, reduction in slow-wave sleep, and increase of time awake in bed. Those changes will eventually make older adults have fragmented sleep and early awakening [5]. In addition to ageing, factors related to insomnia include female sex, low socioeconomic status, comorbid psychiatric or medical 
illness, and depression [6]. Regardless of cultural or ethnic backgrounds, those factors are found to be risk factors for insomnia, despite some conflicting results depending on the countries, cultures, or ethnicities in which the study was conducted.

Studies on insomnia in older adults have focused on the interactions between depression and medical illnesses. Researchers believe that depression and medical illnesses negatively affect each other, which mediated through insomnia [7]. Previous studies have implications of that psychological problems including loneliness, isolation, and worry about the future contribute the mechanisms interaction between depression and insomnia in older adults. The effect of depression on medical illness is mediated by noncompliance and inactivity due to depression is another lesson learned from previous psychosocial research. It is true that these results have contributed in part to the development of treatment strategy in older adults with insomnia and depression. However, there are limitations in understanding insomnia in older adults entirely as a part of the symptoms of depression. Few attempts have made to understand the relationship of emotional regulation with insomnia.

Emotional regulation is extrinsic and intrinsic process about monitoring, evaluation, and controlling the emotional reactions [8]. The studies on emotional regulation of in older adults are inconsistent. Some studies reported that ageing lead to "positivity effect" for emotional regulation, because cognitive decline can make older adults to became less sensitive or more avoidant toward negative information [9]. Meanwhile, another studies found no positivity effect in female older adults [10]. There is a study claiming emotional regulation problem in older adults due to decreased cerebral function related to ageing [11]. Other researchers have reported that that older adults experience more anger or hostility than young adults [12]. Moreover, the findings of that older adults with depression shows more anger expression raise the need to study about the relationship between sleep disturbance and emotional regulation of elderly [13]. Taking that into account, it is necessary to study the relationship of emotional regulation and insomnia among community-dwelling older adults. Hyperarousal model of insomnia also rationalizes these doubts of that emotional regulation problem including anger experience or expression lead to physiological and cognitive hyperarousal, which resulted in insomnia [14].

We hypothesized that the degree of anger experience and expression, which is related to emotional regulation in the older adults, would be associated with insomnia. In order to verify this hypothesis, face-to-face interview and questionnaires about insomnia and anger expression were conducted in the rural community-dwelling older adults. For the assessment of insomnia symptoms, we investigated sleep latency and total sleep time in the participants.

\section{METHODS}

\section{Study population}

This study used the data from "Korean Social life, Health and
Ageing Project (KSHAP)," an ongoing community-based cohort study which aimed to find the interaction between social network and health of Korean older adults in one township ("Y"-myeon, Ganghwa-gun, Incheon, South Korea) [15]. A total of 814 individuals (95\% response rate) were enrolled at the beginning of the study. First wave of the KSHAP study were conducted by face-toface interview in the places suitable for personal conversation including participants' home, or community centers, or public health centers from December 2011 to March 2012. The substudy of relationship between sleep and stress was conducted to participants in the township who agreed to this study (235 individuals, $28.9 \%$ in total study population) from January 2015 to December 2015. This study was approved by the Institutional Review Board of Yonsei University (YUIRB-2011-012-01). All the participants had given explanation of the research purposes and procedure beforehand, and provided written consent voluntarily.

\section{Measurement}

Socio-demographic information (sex, age, education level, job history, current marital status, number of children, number of close relatives or friends, and other social network information) and physical and mental health information (history of medical and psychiatric disease, current psychiatric symptoms including depression, cognitive function) were gathered via face-to-face interview. Participants were asked about perceived economic status (in three categories: better than average, average, worse than average), current smoking and alcohol history.

\section{Structured Clinical Interview for DSM-IV TR Axis I Disorder}

Structured Clinical Interview for DSM-IV TR (SCID) is a research-purpose structured interview that researchers can diagnose psychiatric disorders according to the diagnostic criteria of the Diagnostic and Statistical Manual of Mental Disorders, 4th Edition [16]. In this study, a trained mental health nurse specialist conducted the SCID.

\section{Mini-Mental State Examination for Dementia Screening}

Cognitive function was assessed by the Mini-Mental State Examination for Dementia Screening (MMSE-DS) [17]. MMSE-DS is a modified MMSE which applied the compensatory standard based on age, sex, and education level. It shows better diagnostic accuracy for dementia than MMSE-KC [Mini-Mental Status Examination in the Korean version of the Consortium to Establish a Registry for Alzheimer's Disease (CERAD) assessment packet] [18] or Korean Mini-Mental State Examination (K-MMSE) [19]. We defined cognitive impairment as below-1.5 standard deviation (SD) which was corrected by age and education level [17].

\section{Center for Epidemiologic Studies Depression Scale}

Center for Epidemiologic Studies Depression Scale (CES-D) is a self-reported depression scale consisting of 20 items for the general population, and is a primary screening tool for depressive dis- 
order [20]. Individuals are asked to answer each question from 0 point (very rare, usually less than 1 day in a week) to 3 points (very often, mostly in 5 days or more in a week) depending on frequency and severity of experiences depressive symptoms (depressed feeling, loss of energy, lack of concentration, guilty feeling, feeling of worthlessness, psychomotor retardation, loss of appetite, and sleep disturbance) during the past week. Integrated adaptation of CES-D was used in this study, which is the most recently reported Korean version of the CES-D scale [21]. Current presence of depression is defined as set point of 16 in CES-D scale [22].

\section{Pittsburgh Sleep Quality Index}

Insomnia symptoms of participants was evaluated with the Pittsburgh Sleep Quality Index (PSQI), which is a tool that can assess total sleep time, sleep latency, subjective sleep quality, and freshness [23]. PSQI consists of 7 components including subjective sleep quality, sleep latency, sleep duration, habitual sleep efficiency, sleep disturbances, use of sleeping medication, and daytime dysfunction. We used the question 2 of PSQI ("During the past month, how long has it usually takes you to fall asleep each night?") as sleep latency for the analysis. The component 2 of PSQI (sleep latency) reflects not only the amount of time to sleep but also frequency of the sleep latency. We only focused on the sleep latency amount using the question 2 of PSQI. Total sleep time was defined as question 4 of PSQI ("During the past month, how many hours of actual sleep did you get at night?"). Each component scored from 0 point to 3-point, higher score means worse. In this study, we defined poor sleep quality as the component 1 (subjective sleep quality) of PSQI score $\geq 2$ points. Cronbach alpha of component 1 and 2 calculated from Korean version of PSQI validation study is 0.83 and 0.91 , respectively [23].

\section{Perceived Stress Scale}

Perceived Stress Scale (PSS) is a questionnaire consisting of 10 items that assess the stress experienced by the individual during the past month [24]. Each item scored from 0 point to 4 point, resulting total score ranged from 0 to 40 . Higher total score reflects greater degree perceived stress in PSS. This is not a diagnostic tool; cutoff score is not presented. We defined "severe perceived stress level" as the value of more than 2 SD in PSS. For the analysis,

\section{Korean adaptation of the State-Trait Anger Expression Inventory}

State-Trait Anger Expression Inventory (STAXI) is measuring individual's anger. It is an integrated tool of the previously created state-trait anger scale and anger expression scale. STAXI-K, Korean adaptation of STAXI, measures 5 sub-component, consisted of 20 items asking anger experience (10 items for state-anger, 10 items for trait-anger) and 24 items about three types of anger regulation (anger-in, anger-out, anger-control). Each item rated on 4-points scale. Anger-in refers to internalized anger measured by frequency of repressing or suppressing anger experience, and anger-out refers to externalized anger measured by frequency of how often individuals express anger in other people or things around them.
Lastly, anger-control is measured with the frequency of which an individual attempts to control anger expression [25].

\section{Statistical analyses}

This study analyzed the factors which are clinically significant to determine sleep latency or total sleep time. First, we defined sleep initiation difficulty group as participants who have sleep latency more than 30 minutes, which was measured by the responses to the question 2 of PSQI. We performed $\chi^{2}$-test and independent sample t-test for evaluate sleep initiation difficulty group and control group (Supplementary Table 1).

To develop a model that can predict factors affecting sleep initiation difficulty (sleep latency $>30 \mathrm{~min}$ ), we performed logistic regression analysis with independent variables including the factors that showed significant differences between the sleep initiation difficulty group and control group and the factors which were known to affect insomnia including current psychiatric illness, currently perceived stress, and perceived economic status [26].

Simple regression analysis was performed to evaluate the factors affecting total sleep time. For the next step, multiple regression analysis was performed with a total of 10 independent variables including factors that were found to be significantly correlated with total sleep time from the above simple regression analysis, factors known to have correlations to total sleep time in the previous studies (age and current psychiatric illness), and STAXI-K anger-out score which showed significant correlation with sleep initiation difficulty in this study. Statistical significance was set at $\mathrm{p}<0.05$. All analyses were performed by using SPSS (statistical package for social science) 23 for windows version (IBM Corp., Armonk, NY, USA).

\section{RESULTS}

\section{Factors affecting sleep initiation difficulty}

Socio-demographic and clinical characteristics of participants are shown in Table 1. Female sex, low-education level, presence of depression, poor sleep quality, and shorter total sleep time were more related to the sleep initiation difficulty group than control group (Supplementary Table 1).

Multivariate logistic regression analysis was performed using the factors that showed significant differences in the comparison of the sleep initiation difficulty group and the control group, and the factors mentioned in method that might affect the sleep latency as explanatory variables. As a result, female sex, total sleep time, anger-out score shows significant predictive value for sleep initiation problem (Table 2).

\section{Factors affecting total sleep time}

From simple regression analysis, we found that female sex, perceived low economic status, presence of depression, sleep initiation difficulty, poor sleep quality, and severe perceived stress showed significant correlation with shorter sleep time (Supplementary Table 2). In addition to these factors, age, current psychiatric ill- 
ness, educational level, and STAXI-K anger-out score were used as independent variables for multiple regression analysis. Difficulty in sleep initiation, poor sleep quality, and severe perceived stress were founded as significant variable predicting shorter total sleep time (Table 3).

\section{DISCUSSION}

This study aimed to evaluate the correlation between anger expression and sleep disturbance in community-dwelling older adults who aged 60 years or older. We found that anger expression in

Table 1. Socio-demographic and clinical characteristics of participants

\begin{tabular}{lc}
\multicolumn{1}{c}{ Variables } & Total $(\mathrm{n}=235)$ \\
Age (yrs) & $75.0 \pm 6.22$ \\
Sex, female & $158(67.2)$ \\
Education (yrs) & $2.71 \pm 1.47$ \\
Low education ( $\leq 6$ yrs) & $184(78.3)$ \\
Perceived economic status, lower third & $88(37.4)$ \\
Current job status, no & $86(36.6)$ \\
Current smoking, yes & $14(6.0)$ \\
Current drinking, yes & $41(17.4)$ \\
Psychiatric past history (SCID) & $16(6.8)$ \\
Current psychiatric illness (SCID) & $21(8.9)$ \\
Current depression (CES-D $\geq 16)$ & $61(26.0)$ \\
Interference by pain $(>1 /$ week) & $77(32.8)$ \\
Cognitive impairment (MMSE-DS)* & $40(17.0)$ \\
Sleep initiation difficulty (latency $>30$ min) & $93(39.6)$ \\
Total sleep time (min) & $374.51 \pm 120.47$ \\
Anger out (STAXI-K) & $11.24 \pm 3.57$ \\
Anger in (STAXI-K) & $14.38 \pm 4.20$ \\
Anger control (STAXI-K) & $26.00 \pm 5.31$ \\
Poor sleep quality ( $\geq 2$ of PSQI component 1$)$ & $55(23.4)$ \\
Severe perceived stress (PSS $\geq 27)$ & $8(3.4)$ \\
\hline The values are &
\end{tabular}

The values are means \pm SD or number (percent). ${ }^{*}$ MMSE-DS score was adjusted MMSE score by sex, age, educational level [17]. SCID: Structured Clinical Interview for DSM-IV TR, CES-D: Center for Epidemiological Studies Depression Scale, MMSE-DS: Mini-Mental State Examination for Dementia Screening, STAXI-K: Korean adaptation of the State-Trait Anger Expression Inventory, PSQI: Pittsburgh Sleep Quality Index, PSS: Perceived Stress Scale. older adults was associated with insomnia symptoms. Specifically, sleep initiation difficulty was associated with higher anger expression tendency. On the other hand, total sleep time did not show significant correlation with anger expression tendency.

In this study, sleep initiation difficulty was associated with female sex, anger expression tendency, and shorter total sleep time with considering covariates. Shorter total sleep time was associated with sleep initiation difficulty, severe perceived stress, and poor sleep quality after controlling covariates. These results are in agreement with the findings of previous study conducted in same area but using different time, sample and tools [27]. Findings from the previous study showed that female sex, low educational level, current smoking, comorbid chronic disease, and depression were associated with sleep disturbance. Simple comparison of the results is difficult. Because the previous study measured sleep disturbance with only one question ("Do individual has difficulty with initiating or maintaining sleep?") and did not focus on anger expression tendency. Clinical interpretation of the previous study would be difficult. It is hardly avoidable using one or two questionnaires about sleep in large-scale cohort studies. In this study, we tried to assess sleep quality more thoroughly and in detail by us-

Table 3. Multiple linear regression models of factors associated with total sleep time among community-dwelling older adults

\begin{tabular}{|c|c|c|}
\hline & \multicolumn{2}{|c|}{ Sleep duration } \\
\hline & $\mathrm{B}(\mathrm{SE})$ & $\overline{p \text {-value }}$ \\
\hline Age & $0.433(1.195)$ & 0.717 \\
\hline Female & $-22.546(17.453)$ & 0.198 \\
\hline Low education ( $\leq 6 \mathrm{yrs}$ ) & $11.197(19.311)$ & 0.563 \\
\hline Perceived economic status, lower third & $-26.584(14.653)$ & 0.071 \\
\hline Presence of depression (CES-D $\geq 16$ ) & $-15.598(16.851)$ & 0.356 \\
\hline $\begin{array}{l}\text { Sleep initiation difficulty } \\
\text { (latency }>30 \mathrm{~min} \text { ) }\end{array}$ & $-54.225(15.023)$ & $<0.001$ \\
\hline Anger out (STAXI-K) & $1.402(2.124)$ & 0.510 \\
\hline $\begin{array}{l}\text { Poor sleep quality ( } \geq 2 \text { of PSQI } \\
\text { component } 1 \text { ) }\end{array}$ & $-83.926(17.595)$ & $<0.001$ \\
\hline Severe perceived stress (PSS $\geq 27$ ) & $-83.948(39.074)$ & 0.033 \\
\hline
\end{tabular}

CES-D: Center for Epidemiological Studies Depression Scale, STAXIK: Korean adaptation of the State-Trait Anger Expression Inventory, PSQI: Pittsburgh Sleep Quality Index, PSS: Perceived Stress Scale, SE: standard error.

Table 2. Multiple logistic regression models of factors associated with sleep initiation difficulty among community-dwelling older adults

\begin{tabular}{lccc}
\multicolumn{1}{c}{ Variables } & OR & $95 \%$ CI & p value \\
Age & 1.013 & $(0.964-1.064)$ & 0.609 \\
Female & 2.085 & $(0.996-4.365)$ & 0.051 \\
Low education ( $\leq 6$ yrs) & 1.167 & $(0.513-2.656)$ & 0.712 \\
Perceived economic status, lower third & 1.251 & $(0.689-2.274)$ & 0.462 \\
Current psychiatric illness (SCID) & 1.611 & $(0.600-4.327)$ & 0.344 \\
Presence of depression (CES-D $\geq 16)$ & 1.292 & $(0.665-2.512)$ & 0.450 \\
Total sleep time (min) & 0.995 & $(0.992-0.998)$ & $<0.001$ \\
Anger out (STAXI-K) & 1.122 & $(1.029-1.223)$ & 0.009 \\
Poor sleep quality $(\geq 2$ of PSQI component 1) & 1.106 & $(0.531-2.304)$ & 0.787 \\
\hline
\end{tabular}

SCID: Structured Clinical Interview for DSM-IV TR, CES-D: Center for Epidemiological Studies Depression Scale, STAXI-K: Korean adaptation of the State-Trait Anger Expression Inventory, PSQI: Pittsburgh Sleep Quality Index, OR: odds ratio, CI: confidence interval. 
ing PSQI.

One of the important finding in this study is that anger expression tendency can affect sleep initiation problems. Anger expression tendency was measured by STAXI, which was used to investigate the relationship between emotional regulation and sleep disturbance. STAXI-K assesses how individuals react to anger experience. If individuals experienced anger, they have strategy to react it with three possible ways; anger suppression, anger expression, or anger control. The items measuring anger suppression includes questions such as 'to keep the anger inside' which suggest highly hold back internal anger. The questions such as 'to avoid people while in anger' which can be interpreted as both anger suppression and anger control. There is a possibility that the participants with high anger control tendency respond also positively to items asking anger suppression. As a result, we found that anger expression tendency affects sleep initiation problem. There are scarce of studies on the relationship between anger expression and sleep, and the results are controversial. A study claimed that anger expression is associated with better sleep quality [28]. Another study investigating correlations between anger expression and sleep quality among patients with coronary heart disease using STAXI and PSQI showed that anger suppression is associated with poor sleep quality but anger expression showed positive correlation with sleep quality. A study in juvenile offenders reported that aggression is correlated to sleep quality, especially hostility is negatively affected total sleep time and sleep quality [29]. In addition, a study about reaction to anger with ageing in Korean reported that the anger expression tendency of the older adults is higher than that of the young adults [30].

It is hard to say that anger expression and anger suppression are opposite strategies to react anger. In the STAXI-K validity study in Korea, in contrast to studies conducted in the United States, there is a significant correlation between anger-in and anger-out scores [31]. Therefore, the perspective of that anger expression is opposite side of anger suppression may not be suitable for understanding the mechanism of anger regulation [32]. Rather, the tendency to overuse one side, whether suppression or expression, may be related to emotional arousal. Further study may be needed to investigate the assumption.

The mechanism how emotional arousal associate the brain with sleep disturbance is not fully understood. Cortisol, known as stress hormone, can be a mediator between anger control and sleep regulation. Researchers reported that anger expression is associated with increased cortisol level [33-35]. These studies' results show that anger expression interacts with daily stress to affect cortisol level but anger suppression does not. However, no specific mechanism how cortisol acts in the brain function relevant to sleepwake cycle has been elucidated. There is a neuroimaging study after acute sleep deprivation which reported changes in brain areas including amygdala, insula, ventral striatum, and prefrontal cortex [36]. It suggests that sleep deprivation cause difficulties in emotional regulation by functional alteration of cortical and subcortical structures responsible for emotional regulation. In addition, the possibility that cholinergic system might play a role in dysfunction of the brain structure responsible for emotional regulation is also demonstrated [37]. The cholinergic system is involved in sleep-wake cycle as well. To date, it is suggested that cholinergic system perform mediational function between emotional regulation.

This study has some limitations. First, it is difficult to generalize this finding to all older adults. Second, self-reported measurement such as PSQI, STAXI-K, CES-D could be influenced by the subjective state of participants in evaluation timing, could not measure objectively. Third, we cannot exclude the possibility of that chronic insomnia causes high anger expression tendency. It is known that chronic insomnia itself can cause severe level of stress, which can contribute emotional dysregulation [38]. There is also a previous study about association between difficulty in emotional regulation and long-term sleep deprivation [39]. Thus, the relationship between anger expression and insomnia might be bidirectional. Lastly, the frequency of sleep initiation difficulty was not considered in this study. In PSQI, the component 2 "sleep latency" is sum of the frequency with sleep initiation problem and the amount of the time to fall asleep. We decided to use the data of the question 2 score instead of the component 2 score because of that the participant who showed significant sleep latency problem based on component 2 were too scarce, which can might cause statistical error in analysis.

\section{Supplementary Materials}

The Data Supplement is available with this article at https://doi. org/10.33069/cim.2019.0008.

\section{Acknowledgments}

This work was supported by the Basic Science Research Program through the National Research Foundation of Korea (NRF) funded by the Ministry of Science, ICT \& Future Planning, Republic of Korea (Grant number: 2017R1A2B3008214 to E. Lee); the Korean Mental Health Technology R\&D Project funded by the Ministry of Health \& Welfare, Republic of Korea (Grant number: HM 15C0995 to E. Lee); and the Social Science Korea (SSK) Project through the NRF funded by the Ministry of Education, Republic of Korea (Grant number: 2017S1A3A2067165 to Y. Youm).

\section{Conflicts of Interest}

The authors have no potential conflicts of interest to disclose.

\section{Author Contributions}

Conceptualization: See Woo Kim, Kyung Mee Park, Woo Jung Kim, Suk Kyoon An, Kee Namkoong, Yoosik Youm, Hyeon Chang Kim, Eun Lee. Data curation: See Woo Kim, Kyung Mee Park, Eun Chae Choi, Eun Lee. Formal analysis: See Woo Kim, Kyung Mee Park, Eun Lee. Funding acquisition: Suk Kyoon An, Kee Namkoong, Yoosik Youm, Hyeon Chang Kim, Eun Lee. Investigation: See Woo Kim, Kyung Mee Park, Suk Kyoon An, Kee Namkoong, Yoosik Youm, Hyeon Chang Kim, Eun Lee. Methodology: See Woo Kim, Kyung Mee Park, Eun Lee. Project administration: Yoosik 
Youm, Hyeon Chang Kim, Eun Lee. Resources: Suk Kyoon An, Kee Namkoong, Yoosik Youm, Hyeon Chang Kim, Eun Lee. Software: Eun Chae Choi, Eun Lee. Supervision: Suk Kyoon An, Kee Namkoong, Eun Lee. Validation: See Woo Kim, Kyung Mee Park, Eun Lee. Visualization: See Woo Kim, Kyung Mee Park, Eun Lee. Writing_original draft: See Woo Kim, Kyung Mee Park, Eun Lee. Writing_-review \& editing: Kyung Mee Park, Suk Kyoon An, Kee Namkoong, Yoosik Youm, Hyeon Chang Kim, Eun Lee.

\section{ORCID iDs}

Eun Lee (1)

https://orcid.org/0000-0002-7462-0144

See Woo Kim (1)

https://orcid.org/0000-0002-8635-6096

\section{REFERENCES}

1. Suzuki K, Miyamoto M, Hirata K. Sleep disorders in the elderly: diagnosis and management. J Gen Fam Med 2017;18:61-71.

2. Weyerer S, Dilling H. Prevalence and treatment of insomnia in the community: results from the Upper Bavarian Field Study. Sleep 1991;14:392-398.

3. Schubert CR, Cruickshanks KJ, Dalton DS, Klein BE, Klein R, Nondahl DM. Prevalence of sleep problems and quality of life in an older population. Sleep 2002;25:889-893.

4. Ahn SH, Choi HK, Kim JH, Kim JL. Prevalence of insomnia and associated factors among community-dwelling Korean elderly. J Korean Geriatr Psychiatry 2015;19:32-39.

5. Kamel NS, Gammack JK. Insomnia in the elderly: cause, approach, and treatment. Am J Med 2006;119:463-469.

6. Ancoli-Israel S, Cooke JR. Prevalence and comorbidity of insomnia and effect on functioning in elderly populations. J Am Geriatr Soc 2005;53(7 Suppl):S264-S271.

7. Leggett A, Assari S, Burgard S, Zivin K. The effect of sleep disturbance on the association between chronic medical conditions and depressive symptoms over time. Longit Life Course Stud 2017;8:138-151.

8. Thompson RA. Emotional regulation and emotional development. Educ Psychol Rev 1991;3:269-307.

9. Carstensen LL, Mikels JA. At the intersection of emotion and cognition: aging and the positivity effect. Curr Dir Psychol Sci 2005;14:117-121.

10. Thomsen DK, Mehlsen MY, Viidik A, Sommerlund B, Zachariae R. Age and gender differences in negative affect-is there a role for emotion regulation? Pers Individ Dif 2005;38:1935-1946.

11. Lantrip C, Huang JH. Cognitive control of emotion in older adults: a review. Clin Psychiatry (Wilmington) 2017;3:9.

12. Barefoot JC, Peterson BL, Dahlstrom WG, Siegler IC, Anderson NB, Williams RB Jr. Hostility patterns and health implications: correlates of CookMedley Hostility Scale scores in a national survey. Health Psychol 1991;10: 18-24.

13. Baeg S, Wang SK, Chee IS, Kim SY, Kim JL. Anger in elderly patients with depressive disorders. Psychiatry Investig 2011;8:186-193.

14. Riemann D, Spiegelhalder K, Feige B, Voderholzer U, Berger M, Perlis M, et al. The hyperarousal model of insomnia: a review of the concept and its evidence. Sleep Med Rev 2010;14:19-31.

15. Youm Y, Laumann EO, Ferraro KF, Waite LJ, Kim HC, Park YR, et al. Social network properties and self-rated health in later life: comparisons from the Korean social life, health, and aging project and the national social life, health and aging project. BMC Geriatr 2014;14:102.

16. First MB, Spitzer RL, Gibbon M, Williams JB. Structured clinical interview for DSM-IV-TR axis I disorders, research version, patient edition (SCID-I/ P). New York: Biometrics Research, New York State Psychiatric Institute, 2002.
17. Han JW, Kim TH, Jhoo JH, Park JH, Kim JL, Ryu SH, et al. A normative study of the Mini-Mental State Examination for Dementia Screening (MMSEDS) and its Short form (SMMSE-DS) in the Korean elderly. J Korean Geriatr Psychiatry 2010;14:27-37.

18. Lee JH, Lee KU, Lee DY, Kim KW, Jhoo JH, Kim JH, et al. Development of the Korean version of the Consortium to Establish a Registry for Alzheimer's Disease Assessment Packet (CERAD-K): clinical and neuropsychological assessment batteries. J Gerontol B Psychol Sci Soc Sci 2002;57:P47-P53.

19. Kang YW, Na DL, Hahn SH. A validity study on the Korean Mini-Mental State Examination (K-MMSE) in dementia patients. J Korean Neurol Assoc 1997;15:300-308.

20. Radloff LS. The CES-D scale: a self-report depression scale for research in the general population. Appl Psychol Meas 1997;1:385-401.

21. Chon KK, Choi SC, Yang BC. Integrated adaptation of CES-D in Korea. Korean J Health Psychol 2001;6:59-76.

22. Santor DA, Zuroff DC, Ramsay JO, Cervantes P, Palacios J. Examining scale discriminability in the BDI and CES-D as a function of depressive severity. Psychol Assess 1995;7:131-139.

23. Sohn SI, Kim DH, Lee MY, Cho YW. The reliability and validity of the Korean version of the Pittsburgh Sleep Quality Index. Sleep Breath 2012;16:803812.

24. Baek Y. Korean version of the Perceived Stress Scale-10: development of the scale and exploring the effects of perceied stress on memory [dissertation]. Seoul: Seoul National University; 2010.

25. Chon KK, Hahn DW, Lee CH, Spielberger CD. Korean adaptation of the State-Trait Anger Expression Inventory: anger and blood pressure. Korean J Health Psychol 1997;2:60-78.

26. Kim KW, Kang SH, Yoon IY, Lee SD, Ju G, Han JW, et al. Prevalence and clinical characteristics of insomnia and its subtypes in the Korean elderly. Arch Gerontol Geriatr 2017;68:68-75.

27. Kim WJ, Joo WT, Baek J, Sohn SY, Namkoong K, Youm Y, et al. Factors associated with insomnia among the elderly in a Korean rural community. Psychiatry Investig 2017;14:400-406.

28. Caska CM, Hendrickson BE, Wong MH, Ali S, Neylan T, Whooley MA. Anger expression and sleep quality in patients with coronary heart disease: findings from the Heart and Soul Study. Psychosom Med 2009;71:280-285.

29. Ireland JL, Culpin V. The relationship between sleeping problems and aggression, anger, and impulsivity in a population of juvenile and young offenders. J Adolesc Health 2006;38:649-655.

30. Park JS, Im CS, Kim TS, Wang SK, Chee IS, Kim JL. Trends in anger and aggression across the life span in Korean population. J Korean Geriatr Psychiatry 2010;14:97-103.

31. Chon KK, Hahn DW, Lee CH. Korean adaptation of the State-Trait Anger Expression Inventory (STAXI-K): the case of college students. Korean J Health Psychol 1998;3:18-32.

32. Linden W, Hogan BE, Rutledge T, Chawla A, Lenz JW, Leung D. There is more to anger coping than "in" or "out". Emotion 2003;3:12-29.

33. al'Absi M, Bongard S, Lovallo WR. Adrenocorticotropin responses to interpersonal stress: effects of overt anger expression style and defensiveness. Int J Psychophysiol 2000;37:257-265.

34. Ritsner M, Maayan R, Gibel A, Strous RD, Modai I, Weizman A. Elevation of the cortisol/dehydroepiandrosterone ratio in schizophrenia patients. Eur Neuropsychopharmacol 2004;14:267-273.

35. Steptoe A, Cropley M, Griffith J, Kirschbaum C. Job strain and anger expression predict early morning elevations in salivary cortisol. Psychosom Med 2000;62:286-292.

36. Goldstein AN, Walker MP. The role of sleep in emotional brain function. Annu Rev Clin Psychol 2014;10:679-708.

37. Mu P, Huang YH. Cholinergic system in sleep regulation of emotion and motivation. Pharmacol Res 2019;143:113-118.

38. Kales A, Kales JD. Evaluation and treatment of insomnia. New York: Oxford University Press; 1984.

39. Baum KT, Desai A, Field J, Miller LE, Rausch J, Beebe DW. Sleep restriction worsens mood and emotion regulation in adolescents. J Child Psychol Psychiatry 2014;55:180-190. 
Supplementary Table 1. Characteristics of participants with sleep initiation difficulty

\begin{tabular}{|c|c|c|c|}
\hline & Controls $(n=142)$ & Latency $>30$ minutes $(n=93)$ & p-value \\
\hline Age (yrs) & $75.1 \pm 6.4$ & $74.8 \pm 5.9$ & 0.739 \\
\hline Sex, female & $86(60.6)$ & $72(77.4)$ & 0.007 \\
\hline Low education ( $\leq 6$ yrs) & $105(73.9)$ & $79(84.9)$ & 0.045 \\
\hline Perceived economic status, lower third & $48(34.3)$ & $40(43.0)$ & 0.179 \\
\hline Current job status, no & $48(33.8)$ & $38(40.9)$ & 0.272 \\
\hline Current Smoking, yes & $9(6.3)$ & $5(5.4)$ & 0.761 \\
\hline Current drinking, yes & $26(18.3)$ & $15(16.1)$ & 0.667 \\
\hline Psychiatric past history (SCID) & $10(7.0)$ & $6(6.5)$ & 0.860 \\
\hline Current psychiatric illness (SCID) & $11(7.7)$ & $10(10.8)$ & 0.430 \\
\hline Presence of depression (CES-D $\geq 16$ ) & $12.04 \pm 6.54$ & $14.41 \pm 6.63$ & 0.008 \\
\hline Interference by pain (>1/week) & $46(32.4)$ & $31(33.3)$ & 0.881 \\
\hline Cognitive impairment (MMSE-DS)* & $22(16.3)$ & $18(19.8)$ & 0.501 \\
\hline Total sleep time (min) & $404.99 \pm 110.98$ & $327.99 \pm 120.07$ & $<0.001$ \\
\hline Anger out (STAXI-K) & $10.82 \pm 3.47$ & $11.89 \pm 3.63$ & 0.024 \\
\hline Anger in (STAXI-K) & $14.04 \pm 4.14$ & $14.91 \pm 4.25$ & 0.117 \\
\hline Anger control (STAXI-K) & $26.00 \pm 5.39$ & $25.99 \pm 5.21$ & 0.988 \\
\hline Poor sleep quality ( $\geq 2$ of PSQI component 1 ) & $24(17.0)$ & $31(33.3)$ & 0.004 \\
\hline Severe perceived stress (PSS $\geq 27$ ) & $11.90 \pm 6.71$ & $13.09 \pm 7.60$ & 0.211 \\
\hline
\end{tabular}

The values are means \pm SD or number (percent). ${ }^{*}$ MMSE-DS score was adjusted MMSE score by sex, age, educational level [17]. SCID: Structured Clinical Interview for DSM-IV TR, CES-D: Center for Epidemiological Studies Depression Scale, MMSE-DS: Mini-Mental State Examination for dementia screening, STAXI-K: Korean adaptation of the State-Trait Anger Expression Inventory, PSQI: Pittsburgh Sleep Quality Index, PSS: Perceived Stress Scale. 
Supplementary Table 2. Simple regression analysis of factors associated with total sleep time among community-dwelling older adults

\begin{tabular}{lcc} 
& \multicolumn{2}{c}{ Sleep duration } \\
\cline { 2 - 3 } & B (SE) & p-value \\
Age & $1.214(1.266)$ & 0.339 \\
Sex, female & $-48.664(16.473)$ & 0.003 \\
Low education ( $\leq 6$ yrs) & $-25.963(19.029)$ & 0.174 \\
Perceived economic status, lower third & $-34.584(16.158)$ & 0.033 \\
Current job status, no & $0.545(16.349)$ & 0.973 \\
Current smoking, yes & $55.962(33.069)$ & 0.092 \\
Current drinking, yes & $32.289(20.643)$ & 0.119 \\
Psychiatric past history (SCID) & $32.779(31.191)$ & 0.294 \\
Current psychiatric illness (SCID) & $26.208(27.554)$ & 0.343 \\
Presence of depression (CES-D $\geq 16)$ & $-53.169(17.623)$ & 0.003 \\
Interference by pain (>1/week) & $-6.310(16.774)$ & 0.707 \\
Cognitive impairment (MMSE-DS)* & $-4.120(20.867)$ & 0.844 \\
Sleep initiation difficulty $\quad$ (latency $>30$ min) & $-76.997(15.294)$ & $<0.001$ \\
Anger out (STAXI-K) & & \\
Anger in (STAXI-K) & $0.024(2.211)$ & 0.991 \\
Anger control (STAXI-K) & $-2.431(1.873)$ & 0.196 \\
Poor sleep quality ( $\geq 2$ of PSQI & $-0.961(1.486)$ & 0.518 \\
component 1) & $-108.370(17.160)$ & $<0.001$ \\
Severe perceived stress (PSS $\geq 27)$ & $-133.432(42.540)$ & 0.002 \\
\hline
\end{tabular}

*MMSE-DS score was adjusted MMSE score by sex, age, educational level [17]. SCID: Structured Clinical Interview for DSM-IV TR, CES-D: Center for Epidemiological Studies Depression Scale, MMSE-DS: MiniMental State Examination for dementia screening, STAXI-K: Korean adaptation of the State-Trait Anger Expression Inventory, PSQI: Pittsburgh Sleep Quality Index, PSS: Perceived Stress Scale. 